\title{
SUBHARMONIC FUNCTIONS IN CERTAIN REGIONS
}

\author{
BY \\ JOHN L. LEWIS
}

\begin{abstract}
In a recent paper Hellsten, Kjellberg, and Norstad considered bounded subharmonic functions $u$ in $|z|<1$ which satisfy a certain inequality. They obtained an exact upper bound for the maximum modulus of $u$. We first show that this bound still holds when $u$ satisfies less restrictive hypotheses. We then give an application of this result.
\end{abstract}

1. Introduction. Let $u \leqq 1$ be subharmonic in $\{|z|<1\}$ and put

$$
m(r)=m(r, u)=\inf _{|z|=r} u(z), \quad M(r)=M(r, u)=\max _{|z|=r} u(z)
$$

when $0<r<1$. Recently, Hellsten, Kjellberg, and Norstad [6] have shown that the condition

$$
m(r) \leqq \cos \pi \lambda M(r),
$$

where $\lambda$ is a fixed number in $(0,1)$ and $r$ is arbitrary subject to $0<r<1$, imposes a restriction upon the growth of $M(r)$. In fact they proved the following theorem.

THEOREM A. Let $u \leqq 1$ be subharmonic in $|z|<1$. Let $\lambda$ be a fixed number in $(0,1)$ and suppose that condition (1.1) is satisfied for $0<r<1$. Then there is an extremal subharmonic function

$$
U(z)=\operatorname{Re}\left\{\frac{2}{\pi} \tan \frac{\pi \lambda}{2} \int_{0}^{z} \frac{t^{\lambda-1}-t^{1-\lambda}}{1-t^{2}} d t\right\}, \quad|\arg z| \leqq \pi,
$$

for which (1.1) holds with equality and such that

$$
M(r) \leqq U(r) \leqq \frac{\tan (\pi \lambda / 2)}{\pi \lambda / 2} r^{\lambda},
$$

where the constant is best possible.

We shall consider a condition of type (1.1) under less restrictive hypotheses on $u$. To this end, let $\Omega$ denote a region contained in $|z|<1$, and suppose that

$$
\text { bd } \Omega \cap\{|z|=r\} \neq \varnothing
$$

when $0<r<1$. Here bd $\Omega$ denotes the boundary of $\Omega$. Let $u \leqq 1$ be subharmonic on $\Omega$ and put $M(r)=M(r, u)=\sup _{|z|=r} u(z), M(r)^{+}=\max \{M(r), 0\}$, when $0<r<1$.

Received by the editors June 4, 1970 and, in revised form, May 25, 1971.

AMS 1969 subject classifications. Primary 3115; Secondary 3050.

Key words and phrases. Subharmonic functions, harmonic functions, minimum modulus, maximum modulus, $\cos \pi \lambda$ inequality, growth of the maximum modulus.

Copyright (c) 1972, American Mathematical Society 
Given $\zeta \in$ bd $\Omega$, we write, as in Beurling (see [1, p. 22, b]), $u \rightarrow(\zeta)=\lim \sup _{z \rightarrow \zeta} u(z)$. Furthermore, if $\lim _{z \rightarrow \zeta} u(z)$ exists, we shall denote this limit by $u \leftrightarrow(\zeta)$.

Finally, suppose that $u$ satisfies the condition

$$
u^{\rightarrow}(\zeta) \leqq \cos \pi \lambda M^{+}(|\zeta|)
$$

when $\zeta \in$ bd $\Omega$ and $0<|\zeta|<1$. Here (1.3) corresponds to (1.1). Under this assumption we have

THEOREM 1. Let $u \leqq 1$ be subharmonic in $\Omega$. Let $\lambda$ be a fixed number in $(0,1)$ and suppose that $u$ satisfies (1.3) for each $\zeta$ in bd $\Omega \cap\{0<|z|<1\}$. Then (i) is true whenever $0<r<1$.

Here we remark that the connectivity of $\Omega$ is used in the proof of Theorem 1 only to insure that $\Omega \cap\{|z|=r\} \neq \varnothing, 0<r<1$.

Hence Theorem 1 is valid for any open set $\Omega$ which satisfies (1.2) and the above condition. We shall use this remark in $\S 6$.

Now let $G$ denote an unbounded region in the complex plane $(C)$ and suppose that condition (1.2) with $\Omega=G$ is satisfied whenever $0 \leqq r<\infty$. In case $G=C-(-\infty, 0]$, we shall denote this region by $K$.

Let $h$ be subharmonic in $G$ and satisfy condition (1.3) with $u=h$ whenever $\zeta \in$ bd $G$ and $0<|\zeta|<+\infty$. In addition we assume that $h^{\rightarrow}<+\infty$ at each finite boundary point of $G$. Then as a consequence of Theorem 1 we have

THEOREM 2. If $0<\lambda<1$, and if $h$ satisfies the above hypotheses, then either $h \leqq 0$ or $\lim _{r \rightarrow \infty} M(r) / r^{\lambda}$ exists as a strictly positive or infinite limit.

We note that Heins [5] proved Theorem 2 for the special case $\lambda=\frac{1}{2}$. He also showed for a general $G$ that if $h \leqq 0$, then $\lim _{r \rightarrow \infty} M(r) / r^{1 / 2}$ may not exist. However, if $G=K$ and $h \leqq 0$, then Heins (see [4, pp. 111-114]) proved that $\lim _{r \rightarrow \infty} M(r) / r^{1 / 2}$ $=0$.

We shall consider this question for $0<\lambda<1$. More specifically, let $h$ satisfy the hypotheses of Theorem $2, G=K$, and suppose that $h \leqq 0$. Then if $\frac{1}{2} \leqq \lambda<1$, it follows from Hein's Theorem that $\lim _{r \rightarrow \infty} M(r) / r^{\lambda}=0$, since $M(r) / r^{1 / 2} \leqq M(r) / r^{\lambda} \leqq 0$, for $r>1$. However, if $0<\lambda<\frac{1}{2}$, then $\lim _{r \rightarrow \infty} M(r) / r^{\lambda}$ may not exist, as we show in $\S 7$.

Finally, let $h$ satisfy the hypotheses of Theorem 2, except for (1.3), and suppose that $h\left(z_{0}\right)>0$ for some $z_{0} \in \Omega$. In addition assume that

$$
h^{\rightarrow}(\zeta) \leqq \cos \pi \lambda M(|\zeta|)
$$

when $\zeta \in$ bd $\Omega$ and $0<|\zeta|<\infty$. Then if $0<\lambda<\frac{1}{2}$, we see since $\cos \pi \lambda \geqq 0$, that (1.4) implies (1.3). Hence by Theorem $2, \lim _{r \rightarrow \infty} M(r) / r^{\lambda}>0$. On the other hand, if $\frac{1}{2}<\lambda<1$, then (1.4) is weaker than (1.3), and $\lim _{r \rightarrow \infty} M(r) / r^{\lambda}$ may not exist (see $\S 8$ ). However, if $G$ is suitably restricted, then the conclusion of Theorem 2 is valid under the weaker assumption (1.4) (see §9). 
2. Proof of Theorem 1. The following lemma will play a fundamental role in our proof of Theorem 1.

Lemma 1. Let $u$ and $\Omega$ satisfy the hypotheses of Theorem 1. Then $M(r)^{+}$ $=\max \{M(r), 0\}$ is a nondecreasing convex function of $\log r$ on $(0,1)$.

Proof. First suppose that $\frac{1}{2} \leqq \lambda<1$. Then since $\cos \pi \lambda \leqq 0$, we see from (1.3) that $u^{+}=\max \{u, 0\}$ has a subharmonic extension to $|z|<1$. Hence in this case Lemma 1 is true.

We now prove Lemma 1 for $0<\lambda<\frac{1}{2}$. We proceed by showing

(a) $M(r)^{+}$is nondecreasing,

(b) $M(r)^{+}$is continuous on the right,

(c) $M(r)^{+}$is convex in $\log r$.

Let $0<t<1$ and let $\Omega_{t}=\{|z|<t\} \cap \Omega$. Let $C=\sup _{z \in \Omega_{t}} u(z)$ and put $C^{+}$ $=\max \{C, 0\}$. We claim that

$$
u(z) \leqq \max \left\{\cos \pi \lambda C^{+}, M(t)^{+}\right\}, \quad z \in \Omega_{t} .
$$

Indeed, if $\zeta \in$ bd $\Omega_{t} \cap$ bd $\Omega$ and $\zeta \neq 0$, then

$$
\left(\left.u\right|_{\Omega}(\zeta)\right)^{\rightarrow} \leqq(u(\zeta))^{\rightarrow} \leqq \cos \pi \lambda M(|\zeta|)^{+} \leqq \max \left\{\cos \pi \lambda C^{+}, M(t)^{+}\right\},
$$

thanks to (1.3) and the fact that $\cos \pi \lambda \geqq 0$. Moreover, since $u$ is upper semicontinuous on $\Omega$, we have $u^{\rightarrow} \mid \Omega_{t}(\zeta) \leqq u(\zeta) \leqq M(t)^{+}$when $\zeta \in \Omega \cap$ bd $\Omega_{t}$. Using these facts and the Phragmén-Lindelöf Maximum Principle (see Heins [4, p. 76]), we see that (2.1) is true. Hence, if $0<s<t<1$, then

$$
M(s) \leqq C \leqq \max \left\{\cos \pi \lambda C^{+}, M(t)^{+}\right\},
$$

and so $M(s)^{+} \leqq M(t)^{+}$. This completes the proof of (a).

To prove (b), let $s \in(0,1)$ and note that

$$
M(s) \leqq \lim _{r \rightarrow s^{+}} M(r)^{+}=C_{1} .
$$

If $C_{1}=0$, then (b) is trivially true. Hence we assume that $C_{1}>0$. In this case there exists a sequence $\left(z_{n}\right)_{1}^{\infty}$, where $\left|z_{n}\right|>s$ and $z_{n} \in \Omega$, such that $u\left(z_{n}\right)=M\left(\left|z_{n}\right|\right)$, $\lim _{n \rightarrow \infty} z_{n}=z$, and $|z|=s$, as we see from the inequality:

$$
u^{\rightarrow}(\zeta) \leqq \cos \pi \lambda M(|\zeta|)^{+}<M(|\zeta|),
$$

which is valid for $\zeta \in$ bd $\Omega$ and $s<|\zeta|<1$. Moreover, $z$ is in $\Omega$, since otherwise (1.3) would be contradicted.

Using the above facts and the upper semicontinuity of $u$ at $z$, we obtain $u(z)$ $\geqq \lim _{n \rightarrow \infty} u\left(z_{n}\right)=C_{1}$, and hence, $M(s) \geqq C_{1}$. Since by (2.2) we have $M(s) \leqq C_{1}$, it follows that $M(s)=C_{1}$ and thereupon that (b) is true.

To prove (c), let $0<s<1$ and suppose that $M(s)>0$. We assert that

$$
\lim _{r \rightarrow s^{-}} M(r)^{+}>\cos \pi \lambda M(s) .
$$


Before we prove this assertion, let us use (2.3) to prove (c): Using (2.3), (a), and (b), we see there exist $r_{1}, r_{2}$ such that $0<r_{1}<s<r_{2}<1$ and

$$
M\left(r_{1}\right)>\cos \pi \lambda M\left(r_{2}\right) .
$$

Let $v(z)=u(z)-\cos \pi \lambda M\left(r_{2}\right), r_{1}<|z|<r_{2}$. Then

$$
v \rightarrow(\zeta)=u \rightarrow(\zeta)-\cos \pi \lambda M\left(r_{2}\right) \leqq \cos \pi \lambda\left(M(|\zeta|)-M\left(r_{2}\right)\right) \leqq 0
$$

when $\zeta \in$ bd $\Omega$ and $r_{1}<|\zeta|<r_{2}$. Here we have used (1.3) and (a).

The above inequality implies that $v^{+}=\max \{v, 0\}$ has a subharmonic extension to the annulus $\left\{r_{1}<|z|<r_{2}\right\}$. Hence $M\left(r, v^{+}\right)$is a convex function of $\log r$ on $\left(r_{1}, r_{2}\right)$. Since by (a) and (2.4) we have

$$
M(r, v)=M(r, u)-\cos \pi \lambda M\left(r_{2}, u\right) \geqq M\left(r_{1}, u\right)-\cos \pi \lambda M\left(r_{2}, u\right)>0,
$$

it follows that $M(r, u)$ is convex in $\log r$ on $\left(r_{1}, r_{2}\right)$. Using this fact, (a), and (b), we deduce that (c) is true.

The proof of Lemma 1 for $0<\lambda<\frac{1}{2}$ is now complete, save for (2.3) which we treat as follows: Choose $z_{0} \in \Omega$ such that $\left|z_{0}\right|=s$ and $u\left(z_{0}\right)=M(s)$. This choice is possible since $M(s)>0$ and $u$ satisfies (1.3). Let $\varepsilon>0$ be a small positive number and let $z_{0}+\varepsilon \exp \left(i \theta_{1}\right), z_{0}+\varepsilon \exp \left(i \theta_{2}\right)$ denote the points of intersection of the circle $\left\{\left|z-z_{0}\right|=\varepsilon\right\}$ with the circle $\{|z|=s\}$. We assume, as we may, that $0<\theta_{1}<\theta_{2}$ $<2 \pi$ and $\left\{z_{0}+\varepsilon e^{i \theta}: \theta_{1} \leqq \theta \leqq \theta_{2}\right\} \subset\{|z| \leqq s\}$.

We observe that $\theta_{2}-\theta_{1} \rightarrow \pi$ as $\varepsilon \rightarrow 0$. Using this observation we obtain, if (2.3) is false,

$$
\begin{aligned}
M(s) & =u\left(z_{0}\right) \leqq \frac{1}{2 \pi} \int_{0}^{2 \pi} u\left(z_{0}+\varepsilon e^{i \theta}\right) d \theta \\
& =\frac{1}{2 \pi} \int_{\theta_{1}}^{\theta_{2}} u\left(z_{0}+\varepsilon e^{i \theta}\right) d \theta+\frac{1}{2 \pi} \int_{\theta_{2}}^{\theta_{1}+2 \pi} u\left(z_{0}+\varepsilon e^{i \theta}\right) d \theta \\
& \leqq \cos \pi \lambda M(s)\left(\theta_{2}-\theta_{1}\right) / 2 \pi+M(s+\varepsilon)\left(2 \pi+\theta_{1}-\theta_{2}\right) / 2 \pi \\
& \rightarrow((1+\cos \pi \lambda) / 2) M(s),
\end{aligned}
$$

as $\varepsilon \rightarrow 0$. We have reached a contradiction since $M(s)>0$. Hence (2.3) is true.

3. An associated function. To continue the proof of Theorem 1, we assume, as we may, that equality does not occur in (1.3), since otherwise we consider $u-\varepsilon$ for small $\varepsilon>0$.

In view of (1.3) we see that if $M\left(r_{0}\right)>0,0<r_{0}<1$, then $u$ assumes its maximum at a point $z_{0}=r_{0} \exp \left(i \theta_{0}\right)$ in $\Omega$. Then for fixed $r_{0}$ we may assume that $r_{0} \in \Omega$ and

$$
u\left(r_{0}\right)=M\left(r_{0}\right)
$$

since otherwise we put

$$
\Omega_{1}=\left\{z: z \exp \left(i \theta_{0}\right) \in \Omega\right\}, \quad u_{1}(z)=u\left(z \exp \left(i \theta_{0}\right)\right), \quad z \in \Omega_{1},
$$

and consider $u_{1}$. 
We proceed under these assumptions. Let $v$ be defined in $\{|z|<1\}-\{0\}$ by

$$
v(z)=\max \left\{(1+\cos \pi \lambda) M(|z|)^{+}, u(z)+u(-z)\right\}
$$

when $z$ and $-z$ are in $\Omega$, and by $v(z)=(1+\cos \pi \lambda) M(|z|)^{+}$, otherwise. We assert that $v$ is subharmonic in $\{|z|<1\}-\{0\}$. To verify this assertion note that, by Lemma $1, M(|z|)^{+}$is subharmonic in $\{|z|<1\}-\{0\}$. Hence if $z_{0} \notin \Omega \cup$ bd $\Omega$ or if $z_{0}$ and $-z_{0}$ are in $\Omega$, then $v$ is subharmonic at $z_{0}$.

If $z_{0} \in$ bd $\Omega-\{0\}$, then from (1.3) and Lemma 1 we deduce for $s>0$ and small that $u(z)<\cos \pi \lambda M(|z|)^{+}$when $z \in \Omega \cap\left\{\left|z-z_{0}\right|<s\right\}$. It follows from this inequality that $v(z)=(1+\cos \pi \lambda) M(|z|)^{+},\left|z-z_{0}\right|<s$. Hence in this case $v$ is subharmonic at $z_{0}$.

A similar argument applies if $-z_{0} \notin \Omega$. Hence in all cases $v$ is subharmonic at $z_{0}$ in $\{|z|<1\}-\{0\}$.

Next, we observe, since $v$ is bounded, that $v$ has a subharmonic extension to $\{|z|<1\}$. Indeed, if $h$ is the least harmonic majorant of $v$ in $\{|z|<r\}-\{0\}, 0<r<1$ (see Heins [4, p. 90, ex. 4]), then

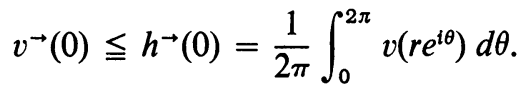

We shall also denote this extension by $v$.

4. A maximum principle. Since $v \leqq 2$ and $v$ is subharmonic in $|z|<1, v$ can be written in the form (see Tsuji [8, IV.10])

$$
v(z)=v_{1}(z)+v_{2}(z)
$$

where

$$
v_{1}(z)=\int_{|\zeta|<1} \log \left|\frac{z-\zeta}{1-\zeta z}\right| d \mu(\zeta)
$$

and

$$
v_{2}(z)=2-\frac{1}{2 \pi} \int_{-\pi}^{\pi} \operatorname{Re} \frac{e^{i \theta}+z}{e^{i \theta}-z} d \alpha(\theta) .
$$

Here $\mu$ is the Riesz mass associated with $v$, and $\alpha$ is a nondecreasing function on $[-\pi, \pi]$ which is continuous on the right and satisfies $\alpha(-\pi)=0 .-v_{1}$ is a Green's potential, and $v_{2}$ is the least harmonic majorant of $v$ in $\{|z|<1\}$.

Since $v(z)=v(-z)$, we have

$$
\begin{aligned}
v(z) & =\frac{v(z)+v(-z)}{2} \\
& =\frac{1}{2} \int_{|\zeta|<1} \log \left|\frac{z^{2}-\zeta^{2}}{1-\zeta^{2} z^{2}}\right| d \mu(\zeta)+2-\frac{1}{2 \pi} \int_{-\pi}^{\pi} \operatorname{Re} \frac{e^{2 i \theta}+z^{2}}{e^{2 i \theta}-z^{2}} d \alpha(\theta) .
\end{aligned}
$$

Let

$$
v^{*}(z)=\frac{1}{2} \int_{|\zeta|<1} \log \left|\frac{z^{2}-|\zeta|^{2}}{1-|\zeta|^{2} z^{2}}\right| d \mu(\zeta)+2-\frac{1}{2 \pi} \operatorname{Re} \frac{1+z^{2}}{1-z^{2}} \int_{-\pi}^{\pi} d \alpha(\theta)
$$


We note that

$$
v^{*}( \pm r) \leqq m(r, v) \leqq M(r, v) \leqq v^{*}( \pm i r),
$$

for $0<r<1$, as is easily verified using the inequalities:

and

$$
\left|\frac{|z|^{2}-|\zeta|^{2}}{1-|\zeta|^{2}|z|^{2}}\right| \leqq\left|\frac{z^{2}-\zeta^{2}}{1-\zeta^{2} z^{2}}\right| \leqq\left|\frac{|z|^{2}+|\zeta|^{2}}{1+|\zeta|^{2}|z|^{2}}\right|
$$

$$
\frac{1-|z|^{2}}{1+|z|^{2}} \leqq \operatorname{Re} \frac{e^{2 i \theta}+z^{2}}{e^{2 i \theta}-z^{2}} \leqq \frac{1+|z|^{2}}{1-|z|^{2}} .
$$

Let $I=\{z: \operatorname{Im} z>0\} \cap\{|z|<1\}$ and $H=\{z: \operatorname{Re} z>0\} \cap\{|z|<1\}$. Let $w$ be the bounded harmonic function in $I$ which has boundary values

$$
\begin{aligned}
w^{\leftrightarrow}( \pm r) & =(1+\cos \pi \lambda) M(r, u)^{+}, & & 0<r<1, \\
w^{\leftrightarrow}\left(e^{i \theta}\right) & =2, & & 0<|\theta|<\pi .
\end{aligned}
$$

Using (4.1) we find that

$$
v^{*}( \pm r) \leqq m(r, v)=(1+\cos \pi \lambda) M(r, u)^{+}=w^{\leftrightarrow}( \pm r)
$$

when $0<r<1$. Since $v^{*} \leqq 2$, it follows that $\left.v^{*}\right|_{I} \leqq w$ and so by (4.1) we have

$$
v(\text { ir }) \leqq v^{*}(\text { ir }) \leqq w(\text { ir }), \quad 0<r<1 .
$$

We now introduce the function $\tilde{v}$ defined in $\{|z|<1\}-\{0\}$ by

$$
\tilde{v}(z)=\max \left\{(1+\cos \pi \lambda) M(|z|, u)^{+}, u(z)+u(\bar{z})\right\}
$$

when $z$ and $\bar{z}$ are in $\Omega$, and by

$$
\tilde{v}(z)=(1+\cos \pi \lambda) M(|z|, u)^{+}
$$

otherwise. We verify in the same way as for $v$ that $\tilde{v}$ is subharmonic in $\{|z|<1\}-\{0\}$.

Let $w_{1}$ be the bounded harmonic function in $H$ with boundary values $w_{1}^{\leftrightarrow}( \pm i r)$ $=w($ ir $), 0<r<1$, and $w_{1}^{\leftrightarrow}\left(e^{i \theta}\right)=2,|\theta|<\pi / 2$. Since $\tilde{v}( \pm i r)=v( \pm i r), 0<r<1$, it follows from (4.2) that $\left.\tilde{v}\right|_{H} \leqq w_{1}$. Hence by (3.1) we have

$$
\tilde{v}\left(r_{0}\right)=2 u\left(r_{0}\right)=2 M\left(r_{0}\right) \leqq w_{1}\left(r_{0}\right) \text {. }
$$

5. An integral inequality. We now proceed as in Hellsten, Kjellberg, and Norstad [6], and Essén (see [3, p. 333]).

Using the Poisson Integral Formula for $I$ we find for $0<t<1$ that

$$
w(i t)=(1+\cos \pi \lambda) \int_{0}^{1} M(s, u)^{+} K(t, s) d s+h(t),
$$

where

and

$$
K(t, s)=(2 t / \pi)\left\{1 /\left(t^{2}+s^{2}\right)-1 /\left(1+t^{2} s^{2}\right)\right\}
$$

$$
h(t)=(8 / \pi) \tan ^{-1}(t)
$$


Likewise,

$$
w_{1}(r)=\int_{0}^{1} w(i t) K(r, t) d t+h(r)
$$

when $0<r<1$.

Inserting (5.1) into (5.2) and interchanging the order of integration, we get

$$
w_{1}(r)=(1+\cos \pi \lambda) \int_{0}^{1} M(s, u)^{+} L(r, s) d s+p(r)+h(r) .
$$

Here $L(r, s)=\int_{0}^{1} K(r, t) K(t, s) d t$ and $p(r)=\int_{0}^{1} h(t) K(r, t) d t, 0<r<1$. Finally, using (4.3) we obtain

$$
2 M\left(r_{0}\right)^{+} \leqq(1+\cos \pi \lambda) \int_{0}^{1} M(s)^{+} L\left(r_{0}, s\right) d s+p\left(r_{0}\right)+h\left(r_{0}\right) .
$$

The above inequality was obtained under the assumption that $M\left(r_{0}\right)>0$. However, (5.3) is still valid if $M\left(r_{0}\right) \leqq 0$, since the right-hand side is always nonnegative. Hence (5.3) is true whenever $0<r_{0}<1$.

(5.3) implies (see Hellsten, Kjellberg, and Norstad [6]) that

$$
M\left(r_{0}\right)^{+} \leqq U\left(r_{0}\right) \leqq \frac{\tan (\pi \lambda / 2)}{\pi \lambda / 2} r_{0}^{\lambda}
$$

Here $U$ is defined as in Theorem A. Since $r_{0}$ is arbitrary subject to $0<r_{0}<1$, the proof of Theorem 1 is complete.

6. Proof of Theorem 2. We now turn to the proof of Theorem 2. Let $h$ and $G$ be as in the statement of this theorem. We assume that $h\left(z_{0}\right)>0$ for some $z_{0} \in G$, since otherwise there is nothing to prove. Let $\left|z_{0}\right|<R<+\infty$ and put $a=\sup _{|z| \leqq R} h(z)$ $>0$. Let

$$
\Omega=\{z: R z \in G\} \cap\{|z|<1\}, \quad u(z)=h(R z) / a, \quad z \in \Omega .
$$

We wish to apply Lemma 1 and Theorem 1 even though our present $\Omega$ need not be a region. To justify this application, we observe that $\Omega$ satisfies (1.2) and the condition $\Omega \cap\{|z|=r\} \neq \varnothing, 0<r<1$. It now follows from the remark after Theorem 1 that we may apply Lemma 1 and Theorem 1 .

In view of Lemma 1 we see that $M(r, u)^{+}=M(r R, h)^{+} / a$ is a nondecreasing convex function of $\log r$ on $(0,1)$. Since $R$ is arbitrary subject to $\left|z_{0}\right|<R<+\infty$, it follows that $a=M(R, h)$. Using this fact and applying Theorem 1 , we obtain

$$
M(r, u)=\frac{M(r R, h)}{M(R, h)} \leqq \frac{\tan (\pi \lambda / 2)}{\pi \lambda / 2} r^{\lambda},
$$

for $0<r<1$. Hence,

$$
\frac{M(r, h)}{r^{\lambda}} \leqq \frac{\tan (\pi \lambda / 2)}{\pi \lambda / 2} \frac{M(R, h)}{R^{\lambda}}, \quad 0<r<R
$$


Letting $R \rightarrow+\infty$ through a suitable sequence in (6.1), we obtain

$$
\frac{M(r, h)}{r^{\lambda}} \leqq \frac{\tan (\pi \lambda / 2)}{\pi \lambda / 2} \liminf _{R \rightarrow \infty} \frac{M(R, h)}{R^{\lambda}}
$$

Let $b=\lim \inf _{R \rightarrow \infty} M(R, h) / R^{\lambda}$. Since $h\left(z_{0}\right)>0$, the above inequality implies $0<b$.

If $b=+\infty$, then Theorem 2 is trivially true. Hence, we assume that $b<+\infty$. Under this assumption, we obtain, by letting $r \rightarrow+\infty$ through a suitable sequence in (6.1), that

$$
\limsup _{r \rightarrow \infty} \frac{M(r, h)}{r^{\lambda}} \leqq \frac{\tan (\pi \lambda / 2) b}{\pi \lambda / 2}<+\infty .
$$

To complete the proof of Theorem 2, we argue as in Essén (see [3, (3.4)]). Let $r_{0} R=x$ and $R s=y$. Changing variables in (5.3) we find that

$$
\frac{2 M(x, h)}{M(R, h)} \leqq \frac{1+\cos \pi \lambda}{R M(R, h)} \int_{0}^{R} M(y, h)^{+} L\left(\frac{x}{R}, \frac{y}{R}\right) d y+p\left(\frac{x}{R}\right)+h\left(\frac{x}{R}\right) .
$$

Multiplying this expression by $x^{-\lambda} M(R, h) / 2$, we get

$$
\begin{aligned}
\frac{M(x, h)}{x^{\lambda}} \leqq & \frac{(1+\cos \pi \lambda)}{2} \int_{0}^{R} \frac{M(y, h)^{+}}{y^{\lambda}}\left\{\left(\frac{y}{x}\right)^{\lambda} \frac{L(x / R, y / R)}{R}\right\} d y \\
& +\left(x^{-\lambda} M(R, h) / 2\right)[p(x / R)+h(x / R)] .
\end{aligned}
$$

The second term on the right-hand side of this inequality approaches zero as $R \rightarrow+\infty$, since lim $\sup _{r \rightarrow \infty} M(r, h) / r^{\lambda}<+\infty$. Using this fact and letting $R \rightarrow+\infty$ in the above inequality, we obtain

$$
\frac{M(x, h)}{x^{\lambda}} \leqq \int_{0}^{\infty} \frac{M(y, h)^{+}}{y^{\lambda}} Q(x, y) d y
$$

where

$$
Q(x, y)=\frac{\left(2(1+\cos \pi \lambda) / \pi^{2}\right)(y / x)^{\lambda} x \log (x / y)}{x^{2}-y^{2}} .
$$

This integral inequality implies by Essén [2] or Kjellberg (see [7, (18)]) that $\lim _{r \rightarrow \infty} M(r, h) / r^{\lambda}$ exists. Since $0<b<+\infty$, we conclude that this limit is positive.

7. Remark. Let $0<\lambda<\frac{1}{2}, G=K=C-(-\infty, 0]$, and suppose that $h \leqq 0$. In this case $\lim _{r \rightarrow \infty} M(r, h) / r^{\lambda}$ may not exist, as we shall show by giving an example.

Let $f$ be an entire function with negative real zeros for which

$$
\liminf _{r \rightarrow \infty} \frac{\log M(r, f)}{r^{2 \lambda}}<\limsup _{r \rightarrow \infty} \frac{\log M(r, f)}{r^{2 \lambda}}<+\infty
$$

Since $f$ has order $<1$, it follows by the Hadamard Factorization Theorem that $f(z)=\prod_{1}^{\infty}\left(1+z / r_{n}\right), z \in C$, where $0<r_{n} \leqq r_{n+1}$.

From the above expression for $f$ we see that $1 \leqq|f(i|z|)| \leqq|f(z)|, \operatorname{Re} z \geqq 0$. Using this inequality and letting $h(z)=-\log \left|f\left(z^{1 / 2}\right)\right|, z \in K$, we find that $h \leqq 0$ and $M(r, h)$ $=h \leftrightarrow(-r)$. Hence (1.3) with $u=h$ is trivially true. 
It is easily shown by a Phragmén-Lindelöf type argument that

$$
\text { If } \lim _{r \rightarrow \infty} \frac{M(r, h)}{r^{\lambda}}=\lim _{r \rightarrow \infty} \frac{h^{\leftrightarrow}(-r)}{r^{\lambda}}=\alpha \text {, then } \lim _{r \rightarrow \infty} \frac{h(r)}{r^{\lambda}}=\frac{\alpha}{\cos \pi \lambda} .
$$

Since $h(r)=-\log M\left(r^{1 / 2}, f\right), 0<r<\infty$, and $f$ satisfies (7.1), we conclude from the contrapositive of $(*)$ that $\lim _{r \rightarrow \infty} M(r, h) / r^{\lambda}$ does not exist.

8. Remark. Let $\frac{1}{2}<\lambda<1$ and suppose that $h$ satisfies the hypotheses of Theorem 2 , save for (1.3). In addition assume that $h$ satisfies (1.4) when $\zeta \in$ bd $\Omega$ and $0<|\zeta|$ $<\infty$. Then in this case $\lim _{r \rightarrow \infty} M(r) / r^{\lambda}$ may not exist, as we show in the following example:

Let $n$ be a nonnegative integer and put

$$
\begin{aligned}
\alpha_{n} & =\left\{2^{n} e^{i \theta}:|\theta| \leqq \pi / 2\right\} & & \text { if } n \text { is even, } \\
& =\left\{2^{n} e^{i \theta}: \pi / 2 \leqq|\theta| \leqq \pi\right\} & & \text { if } n \text { is odd }
\end{aligned}
$$

Let $\mathscr{B}_{n}=\left\{|z|=2^{n}\right\}-\alpha_{n}$. We define $v$ on $C-\bigcup_{0}^{\infty}\left\{ \pm i 2^{n}\right\}$ by

(a) $v$ is continuous in $C-\bigcup_{n=0}^{\infty}\left\{ \pm i 2^{n}\right\}$,

(b) $v(z)=-2^{n} \cos \pi \lambda, z \in \alpha_{n}-\left\{ \pm i 2^{n}\right\}$,

(c) $v(z)=-2^{n}, z \in \mathscr{B}_{n}$,

(d) $v$ is harmonic and bounded in $\left\{2^{n}<|z|<2^{n+1}\right\}$ and in $\{|z|<1\}$.

From the definition of $v$ we see that

$$
\begin{aligned}
v(z)+v(-z)=-\frac{(1+\cos \pi \lambda) 2^{n+1}}{\log 2} \log \left|\frac{z}{2^{n}}\right|-\frac{(1+\cos \pi \lambda)}{\log 2} 2^{n} \log \left|\frac{2^{n+1}}{z}\right| & \\
2^{n} & <|z|<2^{n+1} .
\end{aligned}
$$

Since $v(z)=v(\bar{z})$, it follows that

$$
v( \pm i|z|)=\frac{v(i|z|)+v(-i|z|)}{2} \geqq-(1+\cos \pi \lambda) 2^{n}>-2^{n}
$$

when $2^{n}<|z|<2^{n+1}$.

We shall use (8.1) to show that if $z_{0} \in \mathscr{B}_{n}$, then $v$ is subharmonic at $z_{0}$. To show this, let $E_{n}$ be the component of $\left\{2^{n}<|z|<2^{n+1}\right\}-\{\operatorname{Re} z=0\}$ for which $\mathscr{B}_{n} \subset$ bd $E_{n}$. Then by (8.1) we see that $v \geqq-2^{n}$ on bd $E_{n}-\left\{ \pm i 2^{n}, \pm i 2^{n+1}\right\}$, and so $v \geqq-2^{n}$ in $E_{n}$. Since $v \geqq-2^{n},|z| \leqq 2^{n}$, it follows that $v$ is subharmonic at $z_{0} \in \mathscr{B}_{n}$.

Let $D=C-\left\{\bigcup_{0}^{\infty} \alpha_{n} \cup[-1,0]\right\}$ and put $u=\left.v\right|_{D}$. We wish to construct a subregion $G$ of $D$ such that bd $G \cap\{|z|=r\} \neq \varnothing, 0 \leqq r<+\infty$, and for which $h=\left.u\right|_{G}$ satisfies (1.4).

To construct this region we first observe that

$$
u(z)<\cos \pi \lambda u(-z), \quad 2^{n}<|z|<2^{n+1} .
$$

This observation is verified by comparing the boundary values of both functions 
in $\left\{2^{n}<|z|<2^{n+1}\right\}$. Using (8.2) and the continuity of $M(r, u)$ on $\left(2^{n}, 2^{n+1}\right)$ we see, if $2^{n}<|z|<2^{n+1}$ and $u\left(-z_{0}\right)=M\left(\left|z_{0}\right|, u\right)$, that

$$
u(z)<\cos \pi \lambda M(|z|, u)
$$

when $\left|z-z_{0}\right|<s$ and $s>0$ is small.

The projection on the positive real axis of disks of this type covers any compact subset of $\left(2^{n}, 2^{n+1}\right)$. Hence, if $\varepsilon>0$ is small, there exists a positive integer $k$ and complex numbers $z_{i}, z_{i}^{\prime}(1 \leqq i \leqq k)$ such that

(a) $\left|z_{1}\right|=2^{n}+\varepsilon$ and $\left|z_{k}^{\prime}\right|=2^{n+1}-\varepsilon$,

(b) $\left|z_{i}\right|<\left|z_{i}^{\prime}\right|=\left|z_{i+1}\right|$,

(c) the line segment connecting $z_{i}$ to $z_{i}^{\prime}$ is contained in $\left\{2^{n}<|z|<2^{n+1}\right\}$ and $u$ satisfies (8.3) on it.

Moreover, if $\varepsilon$ is small enough, there exists $z_{0} \in \mathscr{B}_{n}$ and $z_{k+1}^{\prime} \in \mathscr{B}_{n+1}$ such that $u$ satisfies (8.3) when

$$
z \in\left(\left\{\left|z-z_{0}\right|<2 \varepsilon\right\} \cup\left\{\left|z-z_{k+1}^{\prime}\right|<2 \varepsilon\right\}\right) \cap\left\{2^{n}<|z|<2^{n+1}\right\} .
$$

We choose points $z_{0}^{\prime}$ and $z_{k+1}$ in the above sets such that $\left|z_{0}^{\prime}\right|=2^{n}+\varepsilon$ and $\left|z_{k+1}\right|$ $=2^{n+1}-\varepsilon$. Then $u$ satisfies (8.3) on the line segments connecting $z_{i}$ to $z_{i}^{\prime}(0 \leqq i \leqq k+1)$. We remove these segments from $D$.

If this process is carried out in each annulus, then the resulting region $G$ satisfies bd $G \cap\{|z|=r\} \neq \varnothing, 0 \leqq r<+\infty$. Moreover, it follows that $h=\left.u\right|_{G}$ satisfies (1.4) whenever $0<|\zeta|<+\infty$.

Now $M\left(2^{n}, h\right)=-2^{n}$, and $\lim _{r \rightarrow 2^{n}} M(r, h)=-2^{n} \cos \pi \lambda$. Hence,

$$
-\infty=\liminf _{r \rightarrow \infty} \frac{M(r, h)}{r^{\lambda}}<\limsup _{r \rightarrow \infty} \frac{M(r, h)}{r^{\lambda}}=+\infty .
$$

9. Remark. The preceding example shows for $\frac{1}{2}<\lambda<1$ that we may not replace (1.3) in Theorem 2 by (1.4). However, if in addition to (1.2), $G$ satisfies

(*) bd $G \cap\{|z|=r\}$ has Lebesgue angular measure zero for $0<r<\infty$,

then the conclusion of Theorem 2 is still valid under the weaker assumption (1.4). The proof is essentially the same. Here $(*)$ enables us to prove Lemma 1 . The rest of the proof is then unaltered. We omit the details.

Finally, we express our thanks to Professor Bo Kjellberg for several helpful criticisms of this paper.

\section{REFERENCES}

1. A. Beurling, Études sur un problème de majorisation, Thesis, Uppsala, 1933.

2. M. Essén, Note on "A theorem on the minimum modulus of entire functions" by Kjellberg, Math. Scand. 12 (1963), 12-14. MR 28 \#3159.

3. - A generalization of the Ahlfors-Heins theorem, Trans. Amer. Math. Soc. 142 (1969), 331-344. MR 40 \#2894. 
4. M. Heins, Selected topics in the classical theory of functions of a complex variable, Athena Series: Selected Topics in Math., Holt, Rinehart, and Winston, 1962. MR 29 \#217.

5. - Entire functions with bounded minimum modulus; subharmonic function analogues, Ann. of Math. (2) 49 (1948), 200-213. MR 9, 341.

6. U. Hellsten, B. Kjellberg and F. Norstad, Subharmonic functions in a circle, Ark. Mat. 8 (1969), 185-193.

7. B. Kjellberg, $A$ theorem on the minimum modulus of entire functions, Math. Scand. 12 (1963), 5-11. MR 28 \#3158.

8. M. Tsuji, Potential theory, Potential Theory in Modern Function Theory, Maruzen, Tokyo, 1959. MR 22 \#5712.

Department of Mathematics, University of Illinois, Urbana, Illinois 61801

Current address: Department of Mathematics, University of Kentucky, Lexington, Kentucky 40506 